\title{
Glomerular Function in Pima Indians with Noninsulin-dependent Diabetes Mellitus of Recent Onset
}

\author{
Bryan D. Myers, ${ }^{\star}$ Robert G. Nelson," George W. Williams, ${ }^{\ddagger}$ Peter H. Bennett," Sterling A. Hardy," \\ Richard L. Berg," Nicholas Loon, " William C. Knowler," and William E. Mitch" \\ ${ }^{*}$ Division of Nephrology, Stanford University School of Medicine, Stanford, California 94305; ${ }^{\ddagger}$ Department of Biostatistics and \\ Epidemiology, The Cleveland Clinic Foundation, Phoenix, Arizona 85016, and Cleveland, Ohio 44195; ${ }^{8}$ Phoenix Epidemiology and \\ Clinical Research Branch, National Institute of Diabetes and Digestive and Kidney Diseases, Phoenix, Arizona 85014; \\ and "Renal Division, Emory University School of Medicine, Atlanta, Georgia 30322
}

\begin{abstract}
Differential solute clearances were used to characterize glomerular function in 20 Pima Indians with noninsulin-dependent diabetes mellitus (NIDDM) of $<3$ yr duration. 28 Pima Indians with normal glucose tolerance served as controls. In the diabetic group, the glomerular filtration rate (GFR, iothalamate clearance) exceeded the control value by $15 \%(140 \pm 6 \mathrm{vs}$. $122 \pm 5 \mathrm{ml} / \mathrm{min}, P<0.01)$. A corresponding $12 \%$ increase in renal plasma flow (RPF) was not statistically significant and did not account fully for the observed hyperfiltration, suggesting a concomitant elevation of the ultrafiltration pressure or coefficient. The median albumin excretion ratio in NIDDM exceeded control by almost twofold (10.1 vs. $5.8 \mathrm{mg} / \mathrm{g}$ creatinine), a trend which just failed to achieve statistical significance $(P=0.06)$. Fractional clearances of dextrans of broad size distribution were also elevated in diabetic subjects, significantly so for larger dextrans of between 48 and $60 \AA$ radius. A theoretical analysis of dextran transport through a heteroporous membrane revealed glomerular pores in NIDDM to be uniformly shifted towards pores of larger size than in controls. We conclude that an impairment of barrier size selectivity combined with high GFR elevates the filtered protein load in NIDDM of recent onset. We propose that enhanced transglomerular trafficking of protein may predispose to sclerosis of glomeruli in those Pima Indians with NIDDM who ultimately develop diabetic nephropathy. (J. Clin. Invest. 1991. 88:524-530.) Key words: hyperfiltration - barrier size-selectivity - transmembrane protein shunting $\bullet$ heteroporous membrane models
\end{abstract}

\section{Introduction}

Considerable evidence in experimental animals and some indirect evidence in humans suggests that the development of insulin-dependent diabetes mellitus (IDDM) ${ }^{1}$ is accompanied by a

Address correspondence to Bryan D. Myers, M.D., Division of Nephrology, Room S-215, Stanford University Medical Center, Stanford, CA 94305.

Received for publication 19 October 1990 and in revised form 3 April 1991.

1. Abbreviations used in this paper: GFR, glomerular filtration rate; IDDM, insulin-dependent diabetes mellitus; $K_{\mathrm{f}}$, ultrafiltration coefficient; NIDDM, noninsulin-dependent diabetes mellitus; $\Delta P$, transcapillary hydraulic pressure gradient; $r_{0}$, restrictive pore radius; RPF, renal plasma flow; $S$, one standard deviation about $U ; U$, mean pore radius of a log-normal distribution; $\omega_{0}$, fraction of filtrate volume shunted; $\pi_{\mathrm{A}}$, plasma oncotic pressure.

J. Clin. Invest.

(c) The American Society for Clinical Investigation, Inc.

0021-9738/91/08/0524/07 \$2.00

Volume 88, August 1991, 524-530 reduction in renovascular resistance, affecting predominantly preglomerular vessels. An ensuing elevation of the rate and pressure at which glomerular capillaries are perfused results in an abnormally rapid rate of glomerular filtration (GFR) (1-6). It has been hypothesized that glomerular overperfusion and hyperfiltration may predispose to the structural and functional alterations in glomerular capillaries that eventually become clinically manifest as diabetic nephropathy $(7,8)$.

Evidence of comparable hemodynamic changes in the glomerular microcirculation of patients with noninsulin-dependent diabetes mellitus (NIDDM) is sparse and equivocal (9, 10). The paucity of clear information is partly a consequence of the difficulty in defining the onset of NIDDM because of its insidious nature. This difficulty is compounded by the advanced age at which NIDDM develops in most populations. Aging per se has been shown to lower GFR, as have the age-related renovascular occlusive processes associated with atherosclerosis and hypertension (11). Identifying early abnormalities in glomerular function is important because nearly $60 \%$ of endstage renal disease attributed to diabetes has been reported to occur in individuals with NIDDM (12). Thus, a demonstration of glomerular overperfusion and hyperfiltration early in the course of NIDDM would support a potential role for these hemodynamic alterations in the subsequent development of diabetic nephropathy.

The Pima Indians of the Gila River Indian community in Arizona have one of the world's highest incidence rates of NIDDM and, among those who become diabetic, up to $21 \%$ go on to develop diabetic nephropathy (13-15). They provide a unique opportunity to circumvent the foregoing difficulties associated with the evaluation of glomerular function in other populations with NIDDM. In the first place, systematic glucose tolerance testing at intervals of $\sim 2 \mathrm{yr}$ allows the onset of NIDDM in the Pima Indian population to be identified with reasonable accuracy. Secondly, the onset of such NIDDM occurs most frequently in the third through fifth decades of life, thus precluding the need to simultaneously evaluate the effects of advanced aging on the kidney (11). We have accordingly used differential solute clearances to compare glomerular function in a group of Pima Indians with diabetes of recent onset to that in a second group of Pima Indians with normal glucose tolerance.

\section{Methods}

The study was conducted by researchers of a recently formed "Diabetic Renal Disease Study Group." The group is sponsored by the Kidney section of the National Institute of Diabetes and digestive and kidney diseases. It is comprised of scientists of the National Institutes of Health in Phoenix, AZ, members of a renal function research laboratory in the Division of Nephrology at Stanford University, Palo Alto, CA and members of the Department of Biostatistics and Epidemiology of the 
Cleveland Clinic, Cleveland, $\mathrm{OH}$. Review boards of each institution and of the Gila River Indian community approved the protocol and the informed consent procedures that were employed in the present study.

Subject selection. The Gila River Indian Community is inhabited primarily by Pima and the closely-related Tohono-O'odham (Papago) Indians. Those between the ages of 18 and $60 \mathrm{yr}$, whose heritage was at least 50\% Pima, Tohono-O'odham, or a mixture of these two closelyrelated tribes were eligible to participate in this study. Persons found by review of clinical records to have nondiabetic renal disease, those with elevated serum creatinine concentrations $(>1.3 \mathrm{mg} / \mathrm{dl}$ for men, $>1.1$ $\mathrm{mg} / \mathrm{dl}$ for women), and those taking angiotensin-converting enzyme inhibitors were excluded.

Subjects were assigned to diabetic or nondiabetic groups on the basis of oral glucose tolerance tests performed systematically as part of a longitudinal study of diabetes and its complications conducted in the Gila River Indian Community of Arizona since 1965. In accordance with the criteria of the World Health Organization, subjects were diagnosed as having diabetes when the plasma glucose concentration $\geq 200$ $\mathrm{mg} / \mathrm{dl}$ ( $11.1 \mathrm{mmol} / \mathrm{liter}) 2 \mathrm{~h}$ after ingestion of a standardized $75-\mathrm{g} \mathrm{car}-$ bohydrate load (16). To be selected as having diabetes of recent onset, subjects were required to have demonstrated 2 -h postload plasma glucose $<200 \mathrm{mg} / \mathrm{dl}$ at least once within the preceding $36 \mathrm{mo}$ and at all other examinations previous to the one diagnostic for diabetes. Thus, all diabetic subjects were known to have had diabetes for $<3 \mathrm{yr}$.

During the period August 1988 through December 1989, 20 subjects met the foregoing criteria and were enrolled in the diabetic study group. None had ever received insulin therapy, but four were taking an oral hypoglycemic agent. Twenty-eight subjects exhibiting normal glucose tolerance (2-h postload serum glucose $<140 \mathrm{mg} / \mathrm{dl}$ or $7.0 \mathrm{mmol} /$ liter) during the same period volunteered to serve as a nondiabetic control group. Five members of the two groups were receiving treatment with nonsteroidal antiinflammatory agents, and these were withdrawn $7 \mathrm{~d}$ before the clearance study. None of the remaining subjects was receiving any medication that is known to alter glomerular function.

Study protocol. Selected subjects were admitted to the clinical research center in the Phoenix Indian Medical Center on the afternoon before clearance studies, and fasted overnight. Blood pressure was determined the following morning with subjects at rest in the supine position. Systolic and diastolic pressures were measured to the nearest $2 \mathrm{mmHg}$ at the first and fifth Korotkoff sounds. An indwelling plastic cannula was then inserted into the antecubital vein of each arm. Blood was withdrawn from one of these cannulas and assayed for glycosylated hemoglobin $A_{1}$, serum total protein and albumin concentrations, serum creatinine level, and the plasma oncotic pressure. A voided urine sample was collected, whereafter a diuresis was established with an oral water load of $10 \mathrm{ml} / \mathrm{kg}$. Clearance markers were injected through the other indwelling venous cannula, beginning with a loading dose of $30 \%$ iothalamate ( $300 \mathrm{mg}$ plus $3 \mathrm{mg} / \mathrm{kg}$ for each kilogram above $100 \mathrm{~kg}$ ), $20 \%$ paraaminohippuric acid (PAH, $16 \mathrm{mg} / \mathrm{kg}$ ) and $10 \%$ dextran 40 $(150 \mathrm{mg} / \mathrm{kg})$. Each clearance marker was then delivered continuously by an infusion pump. The infusion rates of iothalamate and PAH were calculated to maintain respective plasma concentrations constant at 1.5 and $2.0 \mathrm{mg} / \mathrm{dl}$. Dextran 40 was injected at half the rate of iothalamate. After a 60 -min equilibration period, the bladder was emptied by voiding, and four carefully timed urine collections were made at 20 min intervals. Blood was drawn to bracket each urine collection. To prevent the formation of PAH-glucose adducts in urine, $4 \mathrm{ml}$ of each urine collection was promptly alkalinized by addition of $23 \mu \mathrm{l}$ of $4 \mathrm{M}$ $\mathrm{NaOH}$ (17). The average urinary clearance of iothalamate was equated with the GFR, and the corresponding clearance of PAH with renocortical plasma flow rate (RPF; 18). Fractional clearances $(\theta)$ of dextran macromolecules in the $32-60-\AA$ radius interval were calculated for the first timed collection period by dividing the clearance of a given dextran by that of iothalamate.

Laboratory procedures. Urine specimens obtained before infusion of the clearance markers were promptly frozen and stored at $-70^{\circ} \mathrm{C}$ until the day of albumin and immunoglobulin $\mathrm{G}(\mathrm{IgG})$ assay. A sensi- tive, enzyme-linked immunosorbent assay (ELISA) was used to assay each protein. The ELISA detects human albumin and IgG in a 3-200 $\mathrm{ng} / \mathrm{ml}$ range. By linear regression analysis, the correlation coefficient of the standard curve over this range was 0.999 . After dilution by three to fivefold, urine samples were placed in polystyrene wells coated with buffered antibodies to human albumin or IgG, and each sample was run in triplicate. Goat antihuman albumin or IgG conjugated with alkaline phosphatase was then added (Tago Inc., Burlingame, CA), followed by alkaline phosphatase substrate (Sigma Chemical Co., St. Louis, MO). The color change was measured using an optical densitometer at $490 \mathrm{~nm}$ (Micro-Elisa reader MR 600 A; Dynatech Laboratories, Inc., Alexandria, VA). The intra- and interassay coefficients of variation averaged 9.4 and $10.1 \%$, respectively. The urinary albumin- or IgG-to-urinary creatinine concentration ratio was used as a surrogate measure of the urinary excretion rate of each protein (19-21).

A high-pressure liquid chromatography system with a sensitive ultraviolet light detector was used to assay iothalamate and PAH at 263 nm (model 6A; Instrumentation Shimadzu; Kyoto, Japan). Ultrafiltrates of plasma and diluted urine were injected onto a reverse phase column (\#C18, $5 \mu$ Uiltrasphere; Beckman Instruments Inc., San Ramon, CA). The mobile phase was $1 \%$ acetonitrile and $0.05 \%$ phosphoric acid, and the flow rate $1.5 \mathrm{ml} / \mathrm{min}$. Iothalamate and PAH concentrations were determined from the peak area of each solute, corresponding to column retention times of 10 and $14 \mathrm{~min}$, respectively.

Separation of dextran $\mathbf{4 0}$ in protein-free filtrates of plasma and urine into narrow fractions was achieved by high-pressure liquid chromatography using a pump (model HLC-6A; Instrumentation Shimadzu, Kyoto, Japan) and two Micropak TSK columns in series (SW 3000 and SW 4000; Toyosoda, Tokyo, Japan). The columns were calibrated with five narrowly-dispersed dextran fractions of known molecular weight $(9.9,25.6,40.4,53.3$, and $72.6 \mathrm{kD}$, respectively) kindly provided by $\mathrm{Dr}$. K. Granath of Pharmacia Fine Chemicals (Upsala, Sweden). Dextran concentration was measured using a refractive index detector (model RID-6A; Instrumentation Shimadzu, Kyoto, Japan). An integrator (model 4270, Spectraphysics, San Jose, CA) was used to divide the chromatogram into four slices per minute at a chart speed of 0.25 $\mathrm{cm} / \mathrm{min}$ during the 30-min run. The integrated area of each slice was equated with the dextran concentration at the corresponding retention time. Molecular weight (MW) was calculated from its linear relationship with retention time and molecular radius $\left(r_{\mathbf{s}}\right)$ was then computed from molecular weight using the equation:

$$
r_{\mathrm{s}}=0.33 \times(\mathrm{MW})^{0.463} \text {. }
$$

Oncotic pressure of plasma was determined by membrane osmometry as described previously (22). The concentration of creatinine was determined by a modification of the Jaffe reaction (23). Glycosylated hemoglobin $A_{1}$ was measured by agar gel electrophoresis (24) and serum protein concentration by Coomassie blue binding.

Analysis of glomerular membrane-pore structure. To characterize the size-selective properties of the glomerular barrier in each group of subjects, we employed two theoretical models, each of which represents the glomerular capillary wall as a heteroporous membrane characterized by two pore parameters (25). According to the first of these models, the major portion of the capillary wall is assumed to be perforated by restrictive, cylindrical pores of identical radius $\left(r_{0}\right)$. The model assumes that there exists in addition a parallel "shunt pathway" which does not discriminate among the infused dextrans on the basis of size, and through which passes a small fraction of the filtrate volume. The shunt pathway is characterized by a parameter, $\omega_{0}$, which governs the fraction of filtrate volume passing through the nonrestrictive portion of the membrane (25). The second model represents the glomerular capillary wall as being perforated by cylindrical pores with a continuous log-normal distribution of pore radii (25). The two parameters which characterize this latter representation are the mean pore radius $(U)$ and the standard deviation about the mean $(S)$ of the log-normal distribution of pore sizes. Each model also estimates volume flows and fluxes and protein concentration along the length of the glomerular capillaries, thereby permitting computation of an ultrafiltration coefficient 
$\left(K_{\mathrm{f}}\right)$, which is the product of effective hydraulic permeability and total glomerular capillary surface area in the two human kidneys. The approach used for calculating the intrinsic membrane parameters separates their effects on fractional dextran clearance from those of purely hemodynamic changes (25).

Statistical analysis. Clinical features and some measures of glomerular function in the two groups were compared with an analysis of variance (ANOVA). Results of these variables are presented as means \pm SE. Sex was generally a significant covariate, and ANOVA results were adjusted accordingly. Age was also considered as a covariate but was found to have little effect on group comparisons. Differences in the fractional clearances of dextran at each radius were evaluated with Student's unpaired $t$ test and these results too are presented as means \pm SE. A nonparametric test (Kruskal-Wallis) was used to evaluate differences in the urinary albumin- and IgG-to-creatinine ratios, and results are presented as medians with ranges. Pearson correlation coefficients were used to demonstrate an association between GFR and renal plasma flow, and analysis of covariance was used in a group comparison of GFR adjusting for renal plasma flow. All $P$ values reported are for two-sided tests, and results are deemed significant for $P<0.05$.

\section{Results}

Clinical features. The clinical characteristics of the two groups are summarized in Table I. The 12 men and 16 women with normal glucose tolerance who comprised the control group were aged $37 \mathrm{yr}$ on average. The average age of the eight men and 12 women who comprised the diabetic group was $35 \mathrm{yr}$, and the average duration of NIDDM, as determined by the date of the abnormal glucose tolerance test, was 1 yr. Obesity was common in both groups, with the body mass index averaging $32 \mathrm{~kg} / \mathrm{m}^{2}$ in nondiabetics and $37 \mathrm{~kg} / \mathrm{m}^{2}$ in those with diabetes $(P=0.01)$. Diabetic subjects weighed $14 \mathrm{~kg}$ more than nondiabetics $(P=0.03)$; the men were $24 \mathrm{~kg}$ heavier, and the women $7 \mathrm{~kg}$. No differences in height were observed by sex in either of the groups.

As stated previously, only four members of the diabetic group were receiving treatment with oral hypoglycemic agents. On average, the glycosylated hemoglobin $\mathrm{A}_{1}$ level was elevated to $8.8 \%$ and fasting blood glucose concentration to $190 \mathrm{mg} / \mathrm{dl}$, compared with corresponding values of $5.9 \%$ and $98 \mathrm{mg} / \mathrm{dl}$ in the control group $(P<0.001)$. There were no differences between the two groups with respect to systolic and diastolic arte-

Table I. Clinical Features of the Study Population

\begin{tabular}{lcc}
\hline & Control & NIDDM \\
\hline Men/women & $12 / 16$ & $8 / 12$ \\
Age $(y r)$ & $37.0 \pm 2.4$ & $35.3 \pm 2.4$ \\
Weight $(\mathrm{kg})$ & $86.3 \pm 4.0$ & $100.0 \pm 5.1^{*}$ \\
BMI $\left(\mathrm{kg} / \mathrm{m}^{2}\right)$ & $31.6 \pm 1.4$ & $37.3 \pm 1.6^{*}$ \\
Systolic BP $(\mathrm{mmHg})$ & $118 \pm 3$ & $116 \pm 2$ \\
Diastolic BP $(\mathrm{mmHg})$ & $78 \pm 2$ & $77 \pm 2$ \\
Fasting glucose $(\mathrm{mg} / \mathrm{dl})$ & $98 \pm 3$ & $190 \pm 16^{*}$ \\
Glycosylated hemoglobin $(\%)$ & $5.9 \pm 0.2$ & $8.8 \pm 0.6^{*}$ \\
Serum creatinine $(\mathrm{mg} / \mathrm{dl})$ & $0.86 \pm 0.03$ & $0.73 \pm 0.03^{*}$ \\
Serum total protein $(\mathrm{mg} / \mathrm{liter})$ & $66.6 \pm 0.7$ & $65.5 \pm 1.3$ \\
Serum albumin $(\mathrm{mg} / \mathrm{liter})$ & $40.2 \pm 0.5$ & $38.3 \pm 0.7^{*}$ \\
& & \\
\hline
\end{tabular}

$* P<0.05$.

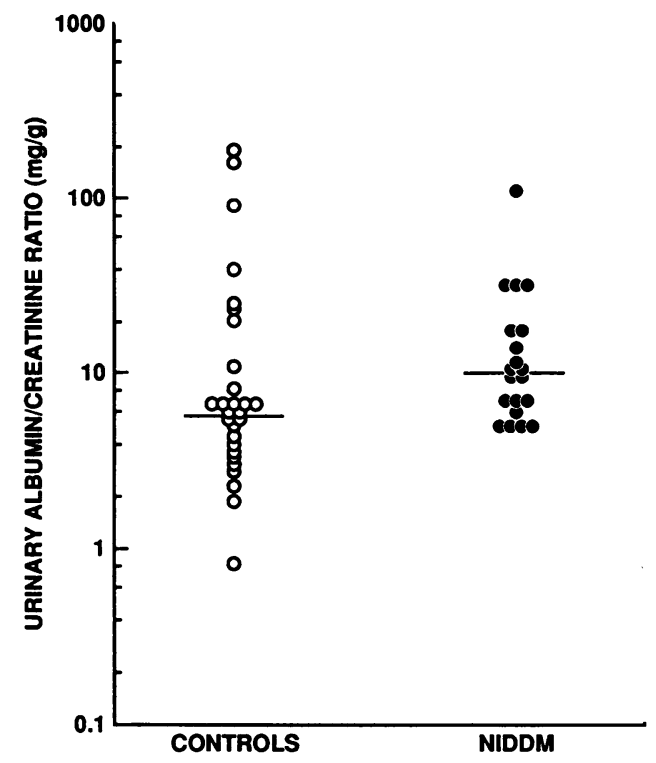

Figure 1. Urinary albumin-to-creatinine excretion ratios in control (left) and diabetic groups (right). The median value is indicated by the horizontal bar.

rial pressures; serum protein concentration was also similar (Table I).

Glomerular function. Although the range of urinary albumin-to-creatinine ratios in subjects with diabetes did not exceed the control range, all but four values in the diabetic group were above the median value of the nondiabetic group (Fig. 1). The excess in the median albumin-to-creatinine ratio in the diabetic group above control narrowly missed achieving statistical significance ( $11 \mathrm{vs.} 6 \mathrm{mg} / \mathrm{g}, P=0.056$ ); the corresponding median IgG-to-creatinine ratios were similar, $5 \mathrm{vs.} 4 \mathrm{mg} / \mathrm{kg}(P$ $=0.31$, Table II).

In keeping with significant depression of the prevailing serum creatinine level $(P=0.003$, Table I) the GFR averaged $140 \pm 6 \mathrm{ml} / \mathrm{min}$ in the diabetic group, $15 \%$ higher than the corresponding value of $122 \pm 5 \mathrm{ml} / \mathrm{min}$ in the control group ( $P$ $=0.01$, Table II). When standard corrections are made for surface area, however, the elevation of GFR in diabetic subjects was by only $7 \%, 119 \mathrm{vs} .110 \mathrm{ml} / \mathrm{min}$ per $1.73 \mathrm{~m}^{2}$, respectively, and statistical significance was no longer achieved $(P=0.20)$. The distribution of GFR, both absolute (Fig. 2) and corrected for body surface area (not shown) reveals the range of values to be the same in both groups. However, most diabetic subjects

Table II. Glomerular Function

\begin{tabular}{lcc}
\hline & Control & NIDDM \\
\hline Albumin excretion ratio $(\mathrm{mg} / \mathrm{g})^{*}$ & $5.8(0.8-184.7)$ & $10.6(4.9-107.0)$ \\
IgG excretion ratio $(\mathrm{mg} / \mathrm{g})^{*}$ & $4.0(0.8-65.3)$ & $4.7(1.5-21.3)$ \\
GFR $(\mathrm{ml} / \mathrm{min})$ & $122 \pm 5$ & $140 \pm 6^{\ddagger}$ \\
PAH clearance $(\mathrm{ml} / \mathrm{min})$ & $618 \pm 25$ & $692 \pm 48$ \\
Filtration fraction & $0.20 \pm 0.01$ & $0.21 \pm 0.01$ \\
$\pi_{\text {A }}(\mathrm{mmHg})$ & $24.3 \pm 0.3$ & $23.6 \pm 0.6$
\end{tabular}

\footnotetext{
${ }^{*}$ Median (range). ${ }^{\ddagger} P<0.05$.
} 


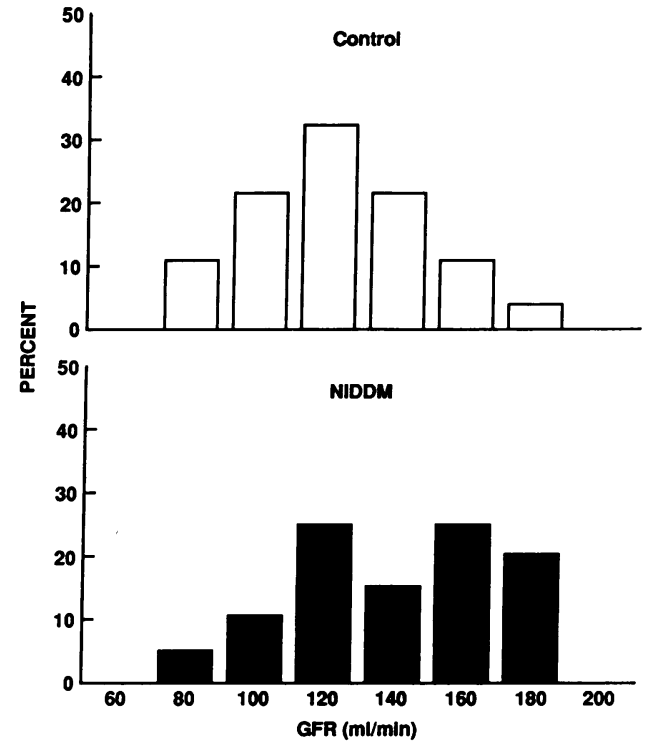

Figure 2. Distribution of GFR in the control group (upper histogram) versus the diabetic group (lower histogram).

had rates in the upper part of the range (Fig. 2). The absolute renal plasma flow rate averaged $692 \pm 48 \mathrm{ml} / \mathrm{min}$ in diabetic subjects, $12 \%$ higher than the $618 \pm 25 \mathrm{ml} / \mathrm{min}$ in controls $(P$ $=0.11)$. Only a $5 \%$ difference remained after correction for body surface area, however $(585 \pm 37$ vs. $560 \pm 25 \mathrm{ml} / \mathrm{min}$ per $\left.1.73 \mathrm{~m}^{2}, P=0.64\right)$. Because obesity per se is not known to influence glomerular filtration and perfusion rates, we have assumed that uncorrected values for GFR and renal plasma flow reflect the diabetic state, and have used these absolute values in the regression analyses and computations that follow.

A positive correlation between GFR and renal plasma flow was found in both groups; the coefficients of correlation were 0.77 and 0.80 in the diabetic and control groups, respectively

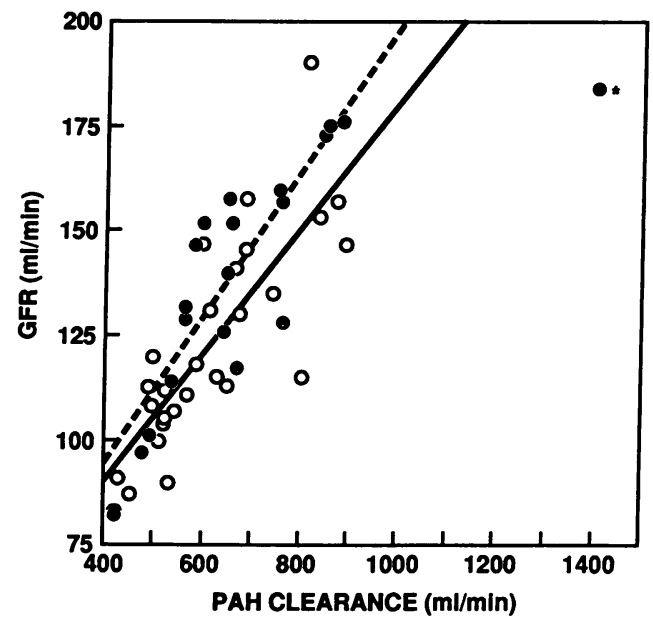

Figure 3. Relationship between GFR and renal plasma flow in the control (0) and diabetic groups (๑). Excluding the outlier in the diabetic group (signified by an asterisk), the equations that describe the slopes for GFR adjusted for renal plasma flow are: GFR $=15.6$ $+0.15 \cdot \mathrm{C}_{\mathrm{PAH}}$ for controls and GFR $=26.3+0.15 \cdot \mathrm{C}_{\mathrm{PAH}}$ for the diabetic group.

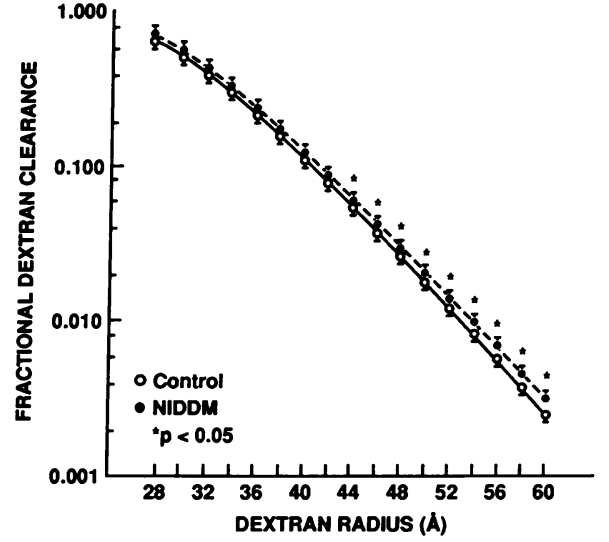

Figure 4. Comparison of the fractional dextran clearance profiles in the diabetic (solid symbols, dashed line) and control groups (open symbols, continuous line). The horizontal bars represent $1 \mathrm{SE}$ for each sieving coefficient. ${ }^{*} P<0.05$ in diabetic vs. control subjects.

$(P<0.001$, Fig. 3). After controlling for renal plasma flow, the mean GFR remained higher in the diabetic than in the control group, 136 vs. $126 \mathrm{ml} / \mathrm{min}$, respectively $(P=0.06)$. Of note, when the single outlier in the diabetic group is excluded (Fig. 3 ), the coefficient of correlation increases to 0.85 , and GFR controlled for renal plasma flow is significantly higher than in the control group $(P=0.02)$. Reflecting significant hypoalbuminemia $(P=0.02$, Table $I)$, plasma oncotic pressure $\left(\pi_{\mathrm{A}}\right)$, a force opposing the rate at which glomerular filtrate is formed, tended to be lower in the diabetic than the control group, $23.6 \pm 0.6$ vs. $24.3 \pm 0.3 \mathrm{mmHg}$, but the difference failed to reach statistical significance $(P=0.11)$.

The mean dextran sieving profiles of the diabetic and control groups are compared in Fig. 4. As shown, fractional dextran clearances in diabetic subjects were uniformly elevated over the entire range of molecular radii tested (32-60 $\AA$ ). The elevation was most marked at the large radius end of the profile, reaching statistical significance for dextrans of $48 \AA$ radius or higher.

Analysis of membrane parameters. The heteroporous membrane models were used to compute representative membrane parameters for each group from the mean dextran sieving profiles illustrated in Fig. 4. These models take the effect of GFR determinants on convective and diffusive transmembrane transport into account, requiring knowledge of GFR, $\mathrm{RPF}, \pi_{\mathrm{A}}$, and the transcapillary hydraulic pressure difference $(\Delta P, 25,26)$. Because the latter quantity cannot be directly measured in humans we have arbitrarily assigned a range of values between 40 and $44 \mathrm{mmHg}$, similar to that observed by micropuncture determinations in rats with diabetes of recent onset $(1,2,8)$. The sieving profile predicted by each model (for $\Delta P=\mathbf{4 0 ~} \mathrm{mmHg}$ ) is compared to that observed in each group in Fig. 5. The predictions of the isoporous + shunt model replicated the observed sieving coefficients at the low radius end of the sieving profile more accurately, whereas those of the lognormal model were more accurate at the high radius end. Judged by a Chi-square analysis, the overall goodness of fit between observed sieving profiles and those predicted by the log-normal model was superior to corresponding predictions by the "isoporous + shunt" model (Tables III and IV).

The computed membrane parameters for the "isoporous 


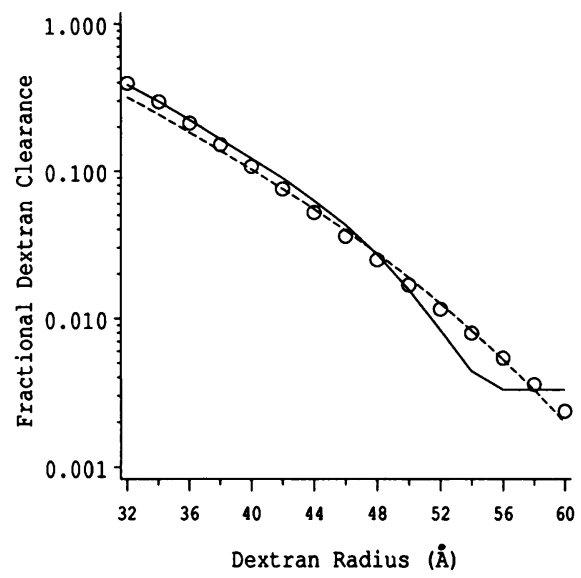

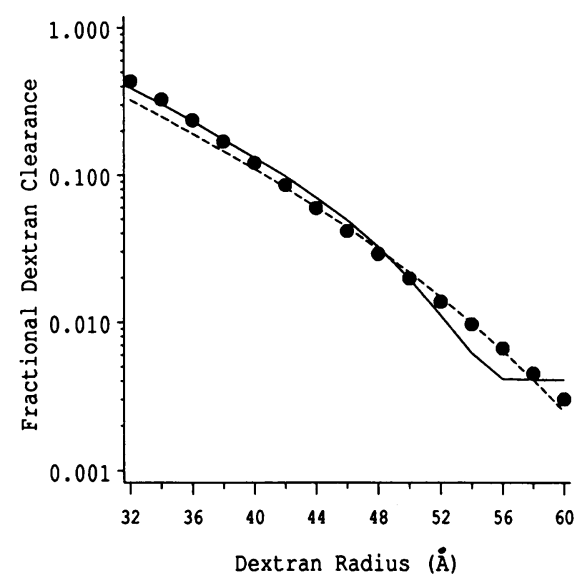

Figure 5. Relationship between predicted and observed sieving coefficients in control (left) and diabetic groups (right). Predictions for the "isoporous + shunt" model (solid curve) and for the log-normal distribution models (dashed curve) are compared to the observed mean sieving coefficients (circles).
+ shunt" model are summarized in Table III. For any given level of $\Delta P$, the computed value for each membrane parameter is higher in the diabetic than in the control group. The elevation of the pore parameters is relatively independent of variations in $\Delta P$. Computed values for $r_{0}$ over the assumed $4 \mathrm{mmHg}$ $\Delta P$ range, vary between 56.6 and $56.7 \AA$ in the diabetic subjects vs. only 55.7-55.8 $\AA$ in controls. A corresponding disparity for $\omega_{0}, 0.0016-0.0020$ in diabetic subjects vs. $0.0012-0.0015$ in controls, suggests that the shunt pathway in the diabetic group is slightly more prominent, regardless of the actual value of $\Delta P$. In contrast, $K_{\mathrm{f}}$, the glomerular ultrafiltration coefficient is strongly dependent on the assumed value of $\Delta P$. As shown in Table III, $K_{\mathrm{f}}$ at any given level of $\Delta P$ is higher in diabetic subjects than controls by $\sim 1 \mathrm{ml} /(\mathrm{min} \cdot \mathrm{mmHg})$. It should be pointed out that according to this model any trend towards an association between diabetes and a higher $K_{\mathrm{f}}$ would be nullified if there was a concomitant increment in $\Delta P$ of only $2 \mathrm{mmHg}$ above any control value in the hypothetical range.

The computed membrane parameters for the continuous lognormal distribution of pore size model are summarized in Table IV, and the calculated distribution of pore radii is illustrated in Fig. 6. The mean radius of the distribution $(U)$ is higher in NIDDM than control, 50.0-50.2 vs. 48.2-48.8 $\AA$. In contrast, the standard deviation, $S$, is essentially 1.15 in each group, indicating that the breadth of the distribution is not altered in NIDDM. Rather, the entire distribution is shifted upwards such that pores of above $60 \AA$ radius, which are those most likely to be permeable to albumin, are more prominent in NIDDM than in the control group (Fig. 6).

Table III. Membrane Parameters (Isoporous + Shunt)

\begin{tabular}{cccccc}
\hline & Assumed $\Delta P$ & $K_{f}$ & $r_{0}$ & $\omega_{0}$ & Sum of $\chi^{2}$ \\
\hline \multirow{6}{*}{ Controls } & $m m H g$ & $m l /(m i n . m m H g)$ & $\dot{A}$ & & \\
& 40 & 11.7 & 55.7 & 0.0012 & 0.749 \\
& 42 & 9.7 & 55.8 & 0.0014 & 0.743 \\
NIDDM & 44 & 8.3 & 55.8 & 0.0015 & 0.753 \\
& 40 & 12.6 & 56.6 & 0.0016 & 0.569 \\
& 42 & 10.6 & 56.6 & 0.0018 & 0.590 \\
& 44 & 9.2 & 56.7 & 0.0020 & 0.621
\end{tabular}

\section{Discussion}

The diabetes mellitus which is so prevalent among Pima Indians is virtually always of the noninsulin-dependent variety, and is frequently complicated by diabetic nephropathy in this population $(13-15,27)$. The diabetic nephropathy exhibits an incidence, course and histopathological appearance, which are essentially indistinguishable from the nephropathy that complicates IDDM $(14,15,28)$. The present study demonstrates yet one more renal abnormality that is common to both IDDM and NIDDM, namely an alteration of glomerular function that can be detected soon after the development of diabetes. The change in glomerular function is characterized by an elevation of absolute GFR and a trend towards enhanced albumin excretion rate. In IDDM each of these abnormalities has been more marked in the presence of uncontrolled hyperglycemia $(4,5)$ and moderated by insulin treatment into a range similar to that observed in the modestly hyperglycemic subjects of the present study $(6,18,29)$. Although measurable hyperfiltration could not be clearly demonstrated in an older population with NIDDM of recent onset, insulin treatment also resulted in a significant depression of GFR under these circumstances (30). Thus, the prevailing GFR appears to be related to the level of glycemia, both in insulin- and noninsulin-dependent diabetes mellitus.

Subtle elevation of the albumin excretion rate into a pathological range detectable only by sensitive immunochemical techniques ( $<300 \mu \mathrm{g} / \mathrm{min}$ ) is referred to as microalbuminuria, and is widely believed to be an early marker of diabetic glomer-

Table IV. Membrane Parameters (Lognormal Distribution)

\begin{tabular}{cccccc}
\hline & Assumed $\Delta P$ & $K_{\mathrm{f}}$ & $U$ & $S$ & Sum of $\chi^{2}$ \\
\hline \multirow{4}{*}{ Controls } & $m m H g$ & $m l /(m i n . m m H g)$ & $\dot{A}$ & & \\
& 40 & 11.8 & 48.4 & 1.154 & 0.186 \\
& 42 & 9.8 & 48.8 & 1.150 & 0.255 \\
NIDDM & 44 & 8.4 & 48.2 & 1.155 & 0.306 \\
& 40 & 12.6 & 50.0 & 1.155 & 0.248 \\
& 42 & 10.6 & 50.1 & 1.146 & 0.304 \\
& 44 & 9.1 & 50.2 & 1.144 & 0.304 \\
& & & & &
\end{tabular}




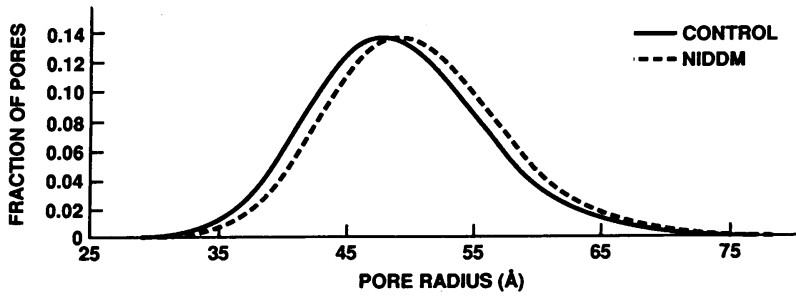

Figure 6. The computed lognormal distribution of pore radii in NIDDM (---) is compared with that of controls (-).

ular injury (31). It is theoretically possible however, that an isolated failure of the tubule to reabsorb a normal load of filtered albumin would result in a rate of albumin excretion in final urine that is within the microalbuminuric range $(32,33)$. Our demonstration of enhanced transglomerular passage of dextrans of broad size distribution documents for the first time that there is a defect in barrier size selectivity in early NIDDM. The presence of such barrier dysfunction suggests that the mechanism for excessive albuminuria in subjects with diabetes of recent onset is enhanced filtration rather than diminished tubular reabsorption of albumin.

Our application of pore theory to the dextran sieving data suggests that enhanced transglomerular passage of dextrans in NIDDM is the consequence of altered membrane-pore structure. Whether the glomerular capillary wall is represented as a membrane perforated by two parallel populations of pores of different radii ("the isoporous + shunt" model), or by a single continuous population of pores with a log-normal distribution of radii, it is evident that recent onset NIDDM is characterized by a generalized increase in pore size. According to the "isoporous + shunt" model, restrictive isopores increase in radius by a little less than $1 \AA$, while shuntlike pores become more prominent. The fraction of filtrate volume by which the shuntlike pores are permeated increases by $25-33 \%$ in the event that $\Delta P$ is unchanged, and by $30-67 \%$ in the event that $\Delta P$ in NIDDM is elevated by $2-4 \mathrm{mmHg}$ (Table III). The generalized increase in pore size can also be appreciated when the second model is used and glomerular pores are represented as a continuous population with a log-normal distribution of radii. In this case, the distribution is shifted upwards with the result that mean pore radius in NIDDM is again larger by just over $1 \AA$ and a more prominent fraction of pores exhibit radii > $60 \AA$ (Fig. 6).

We wish to emphasize that changes in sieving behavior of glomeruli toward dextrans cannot be directly related to the rate at which proteins are excreted in final urine of the subjects of the present study. The prevailing loads of filtered protein in members of both the diabetic and nondiabetic groups are likely to be low, and a substantial but unknown fraction of filtered protein is predicted to undergo tubular reabsorption $(32,33)$. Another confounding factor is that the passage of a given dextran during transglomerular permeation is considerably enhanced compared to that of a rigid protein of similar gel chromatographic radius and molecular charge (34). When this affect of molecular shape is taken into account it is likely that restrictive isopores, which are calculated to present an effective radius of $55 \AA$ to dextran macromolecules, may nevertheless be inpermeable to $36 \AA$ radius albumin. The same is likely to be true of a majority of pores in a continuous log-normal distribution, the mean radius of which approximates only $50 \AA$ A Such shape selectivity should not hinder the passage of albumin through remaining shuntlike pores or pores with radii at the upper end of a log-normal distribution, however. The greater prominence of these very large pores in recent onset NIDDM (Table III, Fig. 6) could thus facilitate the transglomerular passage of albumin and explain the observed trend towards enhanced albuminuria in this circumstance. The absence of a corresponding trend for increased IgG excretion rate would, according to the analysis, reflect the ability of the largest available pores to continue to restrict this large protein, which has a molecular radius of $55 \AA$. Alternatively, fractional tubular reabsorption of IgG at modestly increased filtered loads may be greater than that of albumin. Before endocytosis, filtered proteins bind to the negatively charged glycocalyx of tubule brush border in proportion to their isoelectric points $(35,36)$. Because most species of IgG are neutral or cationic, their reabsorption may be facilitated relative to that of albumin, which is strongly anionic (37).

Our findings provide only limited insights into the mechanism by which GFR became elevated in our diabetic subjects. The strong relationship between GFR and renal plasma flow indicates that the magnitude of the latter quantity contributes to the observed hyperfiltration (Fig. 3). We note with interest, however, that the regression line describing the relationship between GFR and renal plasma flow in the diabetic group is elevated above the corresponding regression line for the nondiabetic group (Fig. 3). Put another way, for any level of renal plasma flow the GFR tends to be higher in diabetic than in nondiabetic subjects. Presumably, an alteration in one or more of the remaining three determinants of GFR must be invoked to explain the observed level of hyperfiltration.

When viewed in physiological terms the GFR can be equated with the product of the net pressure for ultrafiltration and the two-kidney ultrafiltration coefficient, $K_{\mathrm{f}}(38)$. The numerical increase by $12 \%$ in the flow rate of plasma entering the glomerular tufts of our diabetic group (Table II) is predicted to lower the intraluminal concentration of a retained macromolecule as blood flows axially along the glomerular capillaries. Thus, the small but significant depression of albumin concentration in preglomerular plasma (Table I) is likely to become exaggerated within the glomerular capillaries, and to result in an ensuing reduction of glomerular intracapillary oncotic pressure (38). The latter phenomenon in turn should enhance the net pressure for ultrafiltration, thereby favoring a more rapid GFR in NIDDM. Because the changes in dextran passage observed at the low radius end of the sieving profile were small, and because the precision of our theoretical models is limited (Fig. 5), we cannot determine whether concomitant increases in either $\Delta P$ or $K_{\mathrm{f}}$ also served to elevate GFR in the diabetic group (38).

Hyperfiltration, an attendant alteration of glomerular dynamics and enhanced transglomerular trafficking of proteins early in the course of diabetes mellitus have all been proposed as factors which might predispose to the accumulation within glomeruli of extracellular matrix and eventual glomerulosclerosis $(7,8)$. According to this hypothesis the subset of diabetic patients who develop diabetic nephropathy should be those who have marked and sustained glomerular dysfunction from the outset. Our finding of a defect in barrier size selectivity early in the course of NIDDM raises the possibility that a chronic elevation of the filtered protein load may contribute to the pathogenesis of diabetic nephropathy. Increased glomeru- 
lar permeability to protein along with a higher GFR is predicted to increase the rate of protein flux across the glomerular capillary wall. Such increased trafficking of protein in turn could stimulate glomerular cells to overproduce extracellular matrix to the point where surrounding microvessels become obliterated (39). To test this latter hypothesis it will be necessary to demonstrate that both glomerular hyperfiltration and transmembrane trafficking of macromolecules are most pronounced in those subjects who ultimately go on to develop diabetic nephropathy. Our study group has already initiated a longitudinal examination of glomerular function in Pima Indians who develop NIDDM. Results from this study should help to establish whether glomerular dysfunction is sustained in a given individual rather than variable over time. It is our hope that the duration of our study will be sufficiently protracted to establish prospectively whether the magnitude of early glomerular dysfunction is indeed predictive of the ultimate development of diabetic nephropathy.

\section{Acknowledgments}

The authors are indebted to the members of the Gila River Indian community who participated in this investigation, to Lois Jones and Geraldine Swaja who performed, and to Dr. Troy Williams and Dr. Mohammad F. Saad, who helped supervise the clearance studies, and to Kristina Blouch, Karen Schmidt, and Virginia Black who performed the laboratory assays of glomerular function. We also wish to thank Dr. Andrea Remuzzi of the Mario Negri Institute, Bergamo, Italy, for his generous assistance with the theoretical analysis of transglomerular dextran flux.

This work was supported by contract No. N01-DK-7-2291 from the National Institute of Diabetes and Digestive and Kidney Diseases.

\section{References}

1. Hostetter, T. H., J. L. Troy, and B. M. Brenner. 1981. Glomerular hemodynamics in experimental diabetes. Kidney Int. 19:410-415.

2. Scholey, J. W., and T. W. Meyer. 1989. Control of glomerular hypertension by insulin administration in diabetic rats. J. Clin. Invest. 83:1384-1389.

3. Ditzel, J., and M. Schwartz. 1967. Abnormally increased glomerular filtration rate in short-term insulin-treated diabetic subjects. Diabetes. 16:264-267.

4. Christiansen, J. S., J. Gammelgaard, M. Frandsen, and H.-H. Parving 1981. Increased kidney size, glomerular filtration rate and renal plasma flow in short-term insulin-dependent diabetics. Diabetologia. 20:451-456.

5. Morgensen, C. E. 1971. Glomerular filtration rate and renal plasma flow in short-term and long-term juvenile diabetes mellitus. Scand. J. Clin. Lab. Invest. 28:91-100.

6. Hannedouche, T. P., A. G. Delgado, D. A. Gnionsahe, C. Boitard, B. Lacour, and J.-P. Grunfeld. 1990. Renal hemodynamics and segmental tubular reabsorption in early type 1 diabetes. Kidney Int. 37:1126-1133.

7. Hostetter, T. H., H. G. Rennke, and B. M. Brenner. 1982. The case for intrarenal hypertension in the initiation and progression of diabetic and other glomerulopathies. Am. J. Med. 72:375-380.

8. Zatz, R., T. W. Meyer, H. G. Rennke, and B. M. Brenner. 1985. Predominance of hemodynamic rather than metabolic factors in the pathogenesis of diabetic nephropathy. Proc. Natl. Acad. Sci. USA. 82:5963-5967.

9. Schmitz, A. H., T. Christensen, and F. Taagehoej Jenson. 1989. Glomerular filtration rate and kidney volume in normoalbuminuric non-insulin dependent diabetes: lack of glomerular hyperfiltration and renal hypertrophy in uncomplicated NIDDM. Scand. J. Clin. Lab. Invest. 49:103-108.

10. Palmisano, J. J., and H. E. Lebovitz. 1989. Renal function in Black Americans with type II diabetes. J. Diabetic Complications. 3:40-44.

11. Davies, D. F., and N. W. Shock. 1950. Age changes in glomerular filtration rate, effective renal plasma flow, and tubular excretory capacity in adult males. J. Clin. Invest. 29:496-507.

12. Selby, J. V., S. C. Fitzsimmons, J. M. Newman, P. P. Katz, S. Sepe, and J. Showstack. 1990. The natural history and epidemiology of diabetic nephropathy. J. Am. Med. Assoc. 263:1954-1960.

13. Knowler, W. C., P. H. Bennett, R. F. Hamman, and M. Miller. 1978
Diabetes incidence and prevalence in Pima Indians: a 19-fold greater incidence than in Rochester, Minnesota. Am. J. Epidemiol. 108:497-505.

14. Kunzelman, C. L., W. C. Knowler, D. J. Pettitt, and P. H. Bennett. 1989. Incidence of proteinuria in type 2 diabetes mellitus in the Pima Indians. Kidney Int. 35:681-687.

15. Nelson, R. G., C. L. Kunzelman, D. J. Pettitt, M. F. Saad, P. H. Bennett, and W. C. Knowler. 1989. Albuminuria in Type 2 (non-insulin-dependent) diabetes mellitus and impaired glucose tolerance in Pima Indians. Diabetalogia. 32:870-876.

16. World Health Organization. 1985. Diabetes Mellitus. Technical Report Series No. 727. Geneva, Switzerland.

17. Dalton, R. N., M. J. Wiseman, C. Turner, and G. C. Viberti. 1988. Measurement of urinary para-aminohippuric acid in glycosuric diabetics. Kidney Int. 34:117-120.

8. Nyberg G., G. Granerus, and M. Aurell. 1982. Renal extraction ratios for 51 Cr: EDTA, PAH, and glucose in early insulin dependent diabetic patients. Kidney Int. 21:706-708.

19. Ginsberg, J. M., B. S. Chang, R. A. Matarese, and S. Garella. 1983. Use of single voided urine samples to estimate quantitative proteinuria. $N$. Engl. J. Med. 309:1543-1546.

20. Schwab, S. J., R. L. Christensen, K. Dougherty, and S. Klahr. 1987. Quantitation of proteinuria by use of protein-to-creatinine rations in single urine samples. Arch. Intern. Med. 147:943-944.

21. Nathan, D. M., C. Rosenbaum, and V. D. Protasowicki. 1987. Single-void urine samples can be used to estimate quantitative microalbuminuria. Diabetes Care. 10:414-418.

22. Myers, B. D., C. Peterson, C. R. Molina, S. J. Tomlanovich, L. D. Newton, R. Nitkin, H. Sandler, and F. Murad. 1988. Role of cardiac atria in the human renal response to changing plasma volume. Am. J. Physiol. 254:F562673.

23. Shemesh, O., H. Golbetz, J. P. Kriss, and B. D. Myers. 1985. Limitations of creatinine as a filtration marker in glomerulopathic patients. Kidney Int. 28:830-838.

24. Menard, L., M. E. Dempsey, L. A. Blankstein, H. Aleyassine, M. Wacks, and J. S. Soeldner. 1980. Quantitative determination of glycosylated hemoglobin $A_{1}$ by agar gel electrophoresis. Clin. Chem. 26:1598-1602.

25. Deen, W. M., C. R. Bridges, B. M. Brenner, and B. D. Myers. 1985. Heteroporous model of glomerular size selectivity; application to normal and nephrotic humans. Am. J. Physiol. 249:F374-F389.

26. Chang, R. L. S., I. F. Ueki, J. L. Troy, W. M. Deen, C. R. Robertson, and B. M. Brenner. 1975. Permselectivity of the glomerular capillary wall to macromolecules. I. Theoretical considerations. Biophys. J. 15:887-895.

27. Knowler, W. C., P. H. Bennett, G. F. Bottazzo, and D. Doniach. 1979. Islet cell antibodies and diabetes mellitus in Pima Indians. Diabetologia. 17:161164.

28. Kamenetzky, S. A., P. H. Bennett, S. E. Dippe, M. Miller, and P. M. LeCompte. 1974. A clinical and histologic study of diabetic nephropathy in the Pima Indians. Diabetes. 23:61-68.

29. Christiansen, J. S., J. Gammelgaard, B. Tronier, P. A. Svendsen, and H.-H. Parving. 1982. Kidney function and size in diabetics before and during initial insulin treatment. Kidney Int. 21:683-688.

30. Schmitz, A., H. Hvid-Hansen, and T. Christensen. 1989. Kidney function in newly diagnosed Type 2 (non-insulin-dependent) diabetes before and after treatment. Diabetologia 32:434-439.

31. Morgensen, C. E., S. M. Mauer, and C. M. Kjellstrand. 1988. Diabetic nephropathy. In Diseases of the Kidney. 4th ed. R. W. Schrier and C. Gottschalk, editors. Little Brown \& Co., Boston, MA. 2395-2438.

32. Oken, D. E., B. B. Kirschbaum, and D. M. Landwehr. 1981. Micropuncture studies of the mechanisms of normal and pathologic aluminuria. Contrib. Nephrol. 24:1-7.

33. Galaske, R. G., J. B. Van Liew, and L. G. Feld. 1979. Filtration and reabsorption of endogenous low-molecular weight protein in the rat kidney. Kidney Int. 16:394-403.

34. Rennke, H. G., and M. A. Venkatachalam. 1979. Glomerular permeability of macromolecules: effect of molecular configuration on the fractional clearance of uncharged dextran and neutral horseradish peroxidase in the rat. J. Clin. Invest. 63:713-717.

35. Sumpio, B. E., and T. Maack. 1982. Kinetics: competition and selectivity of tubular absorption of proteins. Am. J. Physiol. 243:F379-F392.

36. Christensen, E. L., H. G. Rennke, and F. A. Carone. 1983. Renal tubula uptake of protein: effect of molecular charge. Am. J. Physiol. 244:F436-F441.

37. Nakamura, Y., and B. D. Myers. 1988. Charge selectivity of proteinuria in diabetic glomerulopathy. Diabetes. 37:1202-1211.

38. Deen, W. M., C. R. Robertson, and B. M. Brenner. 1972. A model of glomerular ultrafiltration in the rat. Am. J. Physiol. 223:1178-1183.

39. Mauer, S. M., M. W. Steffes, E. N. Ellis, D. E. R. Sutherland, and D. M. Brown. 1984. Structural-functional relationship in diabetic nephropathy. J. Clin. Invest. 74:1143-1155. 